\title{
Influence of the magnetic configuration on the vortex-lattice instability in $\mathrm{Nb}$ /permalloy bilayers
}

\author{
M. Caputo, ${ }^{1}$ C. Cirillo, ${ }^{1}$ S. Voltan, ${ }^{2}$ A. M. Cucolo, ${ }^{1}$ J. Aarts, ${ }^{2}$ and C. Attanasio ${ }^{1}$ \\ ${ }^{1}$ CNR-SPIN Salerno and Dipartimento di Fisica "E. R. Caianiello”, Università degli Studi di Salerno, \\ via Giovanni Paolo II 132, I-84084 Fisciano (Sa), Italy \\ ${ }^{2}$ Kamerlingh Onnes-Huygens Laboratory, Leiden University, P.O. Box 9504, 2300 RA Leiden, The Netherlands
}

(Received 21 June 2017; revised manuscript received 4 October 2017; published 27 November 2017)

\begin{abstract}
We studied the dynamic instability of the vortex lattice in $\mathrm{Nb} /$ permalloy (permalloy $=\mathrm{Ni}_{0.8} \mathrm{Fe}_{0.2}$ ) bilayers with different Py thickness $d_{\mathrm{Py}}$. When $d_{\mathrm{Py}}$ is larger than some critical value, $d_{\mathrm{cr}}^{\mathrm{ESD}}$, which in our samples has been evaluated to be $\simeq 125 \mathrm{~nm}$, an inhomogeneous magnetic configuration is induced in the samples as a precursor to a fully developed stripe domain state. We found that this inhomogeneous magnetic configuration of the Py layer promotes larger values of the critical velocity for the vortex lattice instability, $v^{*}$, with respect to the homogeneous case.
\end{abstract}

DOI: 10.1103/PhysRevB.96.174519

\section{INTRODUCTION}

Gaining knowledge of the vortex lattice instability and nonequilibrium superconductivity in superconducting/ferromagnetic $(\mathrm{S} / \mathrm{F})$ systems is crucial in the design of $\mathrm{S} / \mathrm{F}$-based devices [1,2]. For example, it was experimentally proved that $\mathrm{S} / \mathrm{F}$ hybrids are optimal systems to be used as ultrafast superconducting optical detectors and eventually even as single photon detectors, due to the small value of the penetration length of the superconducting correlations in the F layer, $\xi_{\mathrm{F}} \lesssim 6 \mathrm{~nm}$, which determines better performance in terms of both photoresponse signal detection and sensitivity [3-5]. In fact, in these devices the light probes a distance $\alpha$ (the material optical penetration depth), which is typically smaller than $50 \mathrm{~nm}$ in the $400-900 \mathrm{~nm}$ visible light wavelength range $[3,4]$. If $\mathrm{S} / \mathrm{N}$ hybrids were to be used (here $\mathrm{N}$ stands for normal metal), due to the value of the penetration length of the Cooper pairs in the $\mathrm{N}$ layer, $\xi_{\mathrm{N}}$, which at low temperatures can be of the order of hundreds of nanometers, a thin $\mathrm{N}$ layer would not be an effective perturbation to the $S$ layer to detect effects induced by the proximity effect. In contrast, in the case of S/F hybrids a thin $\mathrm{F}$ layer will be completely proximized so that different superconducting properties appear at a distance of the order of $\alpha$. Moreover, by studying the dynamic properties of the vortex lattice in $\mathrm{S} / \mathrm{F}$ structures, it was found that the performance of the detecting devices in terms of the quasiparticle relaxation rates seems to be strongly enhanced by the presence of the $F$ layer [1,2,6-8]. In particular, from the analysis of the critical velocity $v^{*}$ for the occurrence of the instability of the moving vortex lattice [9], information on the quasiparticle relaxation time $\tau_{E}$ was obtained, and it was shown that in order to reduce the values of $\tau_{E}$ it is useful to couple the superconductor to a strong ferromagnet, such as permalloy [2]. This conclusion was based on measurements performed on $\mathrm{Nb} / \mathrm{Py}$ bilayers (Py $\equiv$ permalloy $=\mathrm{Ni}_{0.8} \mathrm{Fe}_{0.2}$ ) which showed values of the relaxation time much smaller than that for the single $\mathrm{Nb}$ film [6]. That study was performed on samples with the thickness of the Py layer, $d_{\mathrm{Py}}$, well below the critical thickness $d_{\mathrm{cr}}^{\mathrm{ESD}}$ (ESD stands for emerging stripe-domain phase) where an inhomogeneous magnetic regime can be formed [10].

Indeed, Py shows very interesting magnetic properties. It is well known that when the thickness of a Py film is above a certain critical value $d_{\mathrm{cr}}^{\mathrm{SD}}$ the magnetization of Py is mainly in-plane with a small and alternating out-of-plane component
[11-13]. This is the so-called stripe domain (SD) regime. The exact value of $d_{\mathrm{cr}}^{\mathrm{SD}}$ depends on the fabrication conditions $[12,14,15]$. For our films $d_{\mathrm{cr}}^{\mathrm{SD}} \simeq 300 \mathrm{~nm}$, as mainly determined by magnetic force microscopy (MFM) [10]. Below $d_{\mathrm{cr}}^{\mathrm{SD}}$ two magnetic regimes are present: a homogeneous $(\mathrm{H})$ regime up to $d_{\mathrm{Py}}=d_{\mathrm{cr}}^{\mathrm{ESD}} \simeq 0.5 d_{\mathrm{cr}}^{\mathrm{SD}}$, and a new regime, for $d_{\mathrm{cr}}^{\mathrm{ESD}} \lesssim d_{\mathrm{Py}} \lesssim$ $d_{\mathrm{cr}}^{\mathrm{SD}}$, which was called the emerging stripe-domain (ESD), where a certain degree of magnetic inhomogeneities is present in Py, even though the magnetization is not yet arranged in stripes. In this regime, MFM, for instance, does not detect variations in the magnetic structure. This magnetic regime has been found to be more developed in microstructured samples [10]. Thus, both the ESD and the SD regimes are characterized, although in different forms, by an inhomogeneous magnetic configuration. It was recently observed [16] that, when it is in the ESD regime, Py can generate a long-range proximity effect, probably of spin-triplet nature $[17,18]$.

In this paper, we analyze the dynamic instability of the moving vortex lattice in $\mathrm{Nb} / \mathrm{Py}$ bilayers with $d_{\mathrm{Py}}$ varied across the $\mathrm{H}, \mathrm{ESD}$, and SD regimes. The aim is to investigate the possible influence of the magnetic configuration of the ferromagnetic layer on the instability of the moving vortex lattice, thereby finding further evidence for the presence of the ESD phase. This fact could in principle affect also the values of the relaxation time and, eventually, improve the performance of a radiation detector based on $\mathrm{Nb} / \mathrm{Py}$ heterostructures. We found that in the bilayers prepared both in the ESD and SD regimes the instability sets in at larger values of the vortex velocity with respect to the $\mathrm{H}$ regime.

The paper is organized as follows. In Sec. II we describe the fabrication and the measurement methods and the preliminary characterization of the samples. Section III deals with the analysis of the main experimental data. Finally, in Sec. IV the obtained results are discussed.

\section{EXPERIMENT}

$\mathrm{The} \mathrm{Nb} / \mathrm{Py}$ bilayers were deposited in an ultrahigh vacuum dc diode magnetron sputtering system on $\mathrm{Si}(100)$ substrates kept at room temperature. The base pressure was $2.7 \times 10^{-8}$ mbar, while the deposition was realized in an Ar pressure of $2.7 \times 10^{-3}$ mbar. The bilayers have the same $\mathrm{Nb}$ thickness, $d_{\mathrm{Nb}}=30 \mathrm{~nm}$, and the Py thicknesses $d_{\mathrm{Py}}=50,200$, and 
TABLE I. Characteristic parameters of the measured samples. $T_{\mathrm{c}}$ is the critical temperature at zero magnetic field, $\rho_{\mathrm{n}}$ is the resistivity at $T \simeq 10 \mathrm{~K}, \mu_{0} H_{\mathrm{c} 2 \perp}(t=0)\left[\mu_{0} H_{\mathrm{c} 2 \perp}(t=0.5)\right]$ is the perpendicular upper critical field at the reduced temperature, $t$, equal to $0(0.5)$.

\begin{tabular}{lcccr}
\hline \hline Sample & $T_{\mathrm{c}}(\mathrm{K})$ & $\rho_{\mathrm{n}}(\mu \Omega \mathrm{cm})$ & $\mu_{0} H_{\mathrm{c} 2 \perp}(t=0)(\mathrm{T})$ & $\mu_{0} H_{\mathrm{c} 2 \perp}(t=0.5)(\mathrm{T})$ \\
\hline $\mathrm{Nb}$ & 6.32 & 24.0 & 4.03 & 2.01 \\
$\mathrm{Nb} /$ Py50 & 5.15 & 15.6 & 2.66 & 1.33 \\
$\mathrm{Nb} /$ Py200 & 5.08 & 13.9 & 2.16 & 1.06 \\
$\mathrm{Nb} /$ Py350 & 5.10 & 13.3 & 2.59 & 1.29 \\
\hline \hline
\end{tabular}

$350 \mathrm{~nm}$, in order to explore the influence of the three magnetic regimes, $\mathrm{H}, \mathrm{ESD}$, and $\mathrm{SD}$, on the dynamics of the vortex lattice. For comparison, a single $\mathrm{Nb}$ film $30 \mathrm{~nm}$ thick was also studied. All the samples were patterned by $e$-beam lithography followed by Ar-ion etching into bridges with width $W=$ $10 \mu \mathrm{m}$ and distance between the voltage contacts $L=800 \mu \mathrm{m}$. Electric transport measurements were performed in a ${ }^{4} \mathrm{He}$ cryostat using a standard dc four-probe technique. To minimize the heating effects, the samples were always immersed in liquid helium, confining the measurements to $4.2 \mathrm{~K}$ or below. To measure the $I-V$ characteristics the current biasing was realized by sending rectangular current pulses to the sample, with the current-on time of $12 \mathrm{~ms}$ followed by a current-off time of $1 \mathrm{~s}$. When the current was swept upward and then downward no hysteresis was detected in the $I-V$ curves. This gives a first indication that the instability observed in the curves has no thermal origin. The magnetic field $H$ was applied perpendicularly to the plane of the substrate. For the bilayers $\mathrm{Nb} / \mathrm{Py} 200$ and $\mathrm{Nb} / \mathrm{Py} 350$ (here the numbers indicate the Py thickness expressed in nanometers) the $I-V$ characteristics were measured before and after inducing the inhomogeneous magnetic phase in the Py layers: the ESD and SD regimes, respectively. These phases were obtained by applying a strong magnetic field $(\sim 2 \mathrm{~T})$ perpendicular to the bridge [11], which was removed before starting the measurements. We call the state of the sample before inducing inhomogeneities the virgin (V) state. The temperature dependence of the perpendicular upper critical magnetic field, $H_{\mathrm{c} 2 \perp}(T)$, was obtained by measuring both $R(T)$ and $R(H)$ curves. $T_{\mathrm{c}}$ was defined as the temperature at which the resistivity was equal to $10 \%$ of $\rho_{\mathrm{n}}$, the low-temperature normal state resistivity. The $\mathrm{Nb}$ reference sample has a critical temperature and a low-temperature resistivity of $T_{\mathrm{c}}=6.32 \mathrm{~K}$ and $\rho_{\mathrm{n}}=24.0 \mu \Omega \mathrm{cm}$, respectively. From $\mu_{0} d H_{c 2 \perp} / d T=-0.63 \mathrm{~T} / \mathrm{K}$ close to $T_{\mathrm{c}}$ of the single $\mathrm{Nb}$ film it was possible to evaluate the quasiparticle diffusion coefficient [19] $D=\left(4 k_{\mathrm{B}} / \pi e\right) \times\left(-\mu_{0} d H_{\mathrm{c} 2 \perp} /\left.d T\right|_{T=T_{\mathrm{c}}}\right)^{-1}=$ $1.74 \times 10^{-4} \mathrm{~m}^{2} / \mathrm{s}$. Assuming a linear behavior of the temperature dependence of the perpendicular upper critical field, it is also possible to evaluate the Ginzburg-Landau coherence length at $T=0, \xi_{\| 0}$, as $\mu_{0} H_{c 2 \perp}(0)=\Phi / 2 \pi \xi_{\| 0}^{2}$, where $\Phi$ is the magnetic flux quantum. This results in $\xi_{\| 0}=9.1 \mathrm{~nm}$, which gives a superconducting coherence length $\xi_{\mathrm{s}}=2 \xi_{\mid 0} / \pi=$ $5.8 \mathrm{~nm}$. Moreover, from the values of $\rho_{\mathrm{n}}$ the low-temperature mean free path was extracted by using the expression [20] $\rho \ell=\left(1 / v_{\mathrm{Nb}} \gamma_{\mathrm{Nb}}\right)\left(\pi k_{\mathrm{B}} / e\right)^{2}$, with the values for the electronic specific heat coefficient $\gamma_{\mathrm{Nb}} \cong 7 \times 10^{2} \mathrm{~J} / \mathrm{K}^{2} \mathrm{~m}^{3}$ and the Fermi velocity $v_{\mathrm{Nb}}=2.73 \times 10^{7} \mathrm{~cm} / \mathrm{s}$ [21,22]. This results in $\ell=1.2 \mathrm{~nm} \ll \xi_{\| 0}$, which indicates that the $\mathrm{Nb}$ is in the dirty limit regime. Table I summarizes some characteristic parameters of the investigated samples. In particular, the values of $\rho_{\mathrm{n}}$ of the bilayers show that a good metallic coupling is present between the layers. In fact, if Py resistivity $\rho_{\mathrm{Py}}$ is calculated assuming valid a parallel resistor model and using the measured value of the resistivity of the $30-\mathrm{nm} \mathrm{Nb}$ film, we obtain $\rho_{\mathrm{Py}} \approx 35 \mu \Omega \mathrm{cm}$, consistent with the values of resistivity of single Py films [16].

\section{RESULTS}

In Fig. 1 we present the low-voltage region of the $I-V$ characteristics for the single $\mathrm{Nb}$ film for different values of the magnetic field at the reduced temperature $t=T / T_{\mathrm{c}}=0.5$. The inset shows the curves in the entire measurement range, up to the normal state resistance. Not visible in the main figure, at very low currents, just above the critical current $I_{c}$ the curves exhibit a nonlinear behavior associated with flux creep or plastic motion of the vortex lattice. When the bias current is further increased, the linear flux-flow regime is present until a certain value $I^{*}$, where a sudden jump of the voltage $V^{*}$ takes place in agreement with the Larkin-Ovchinnikov theory [9]. Such instabilities were previously observed in a number of different superconducting systems $[1,6,7,23-38] . V^{*}$ is related to the so-called critical velocity $v^{*}$ by the relation [9]

$$
V^{*}=\mu_{0} v^{*} H L \text {. }
$$

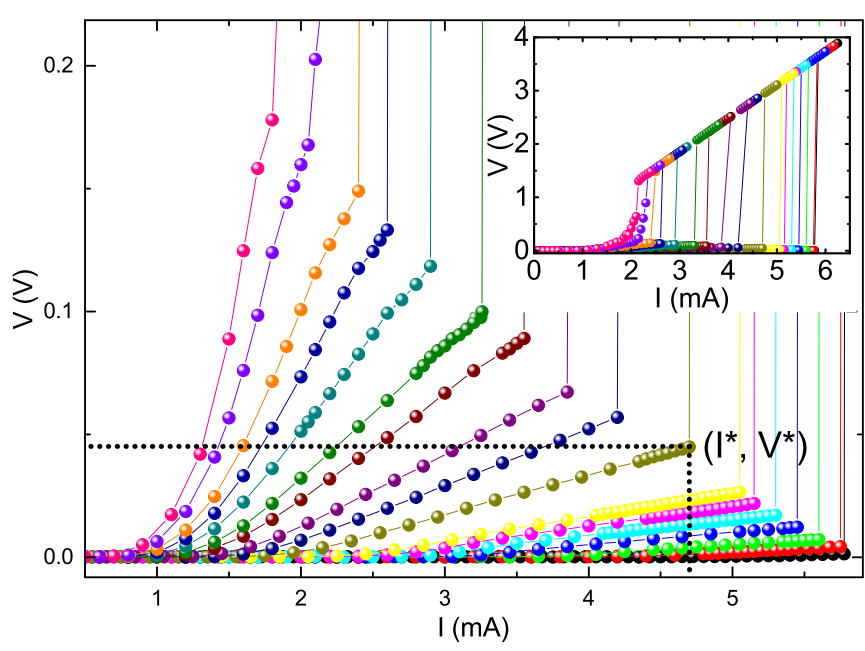

FIG. 1. Low-voltage region of the $I-V$ curves for the $\mathrm{Nb}$ film at $t=0.5$ for different applied magnetic fields. From right to left, the curves correspond to fields of $0.005,0.007,0.01,0.015,0.02,0.025$, $0.03,0.05,0.07,0.09,0.125,0.15,0.2,0.25,0.3,0.4,0.5$ T. $I^{*}$ and $V^{*}$ are indicated for the sake of clarity. Inset: full scale view of the same $I-V$ curves shown in the main panel. 

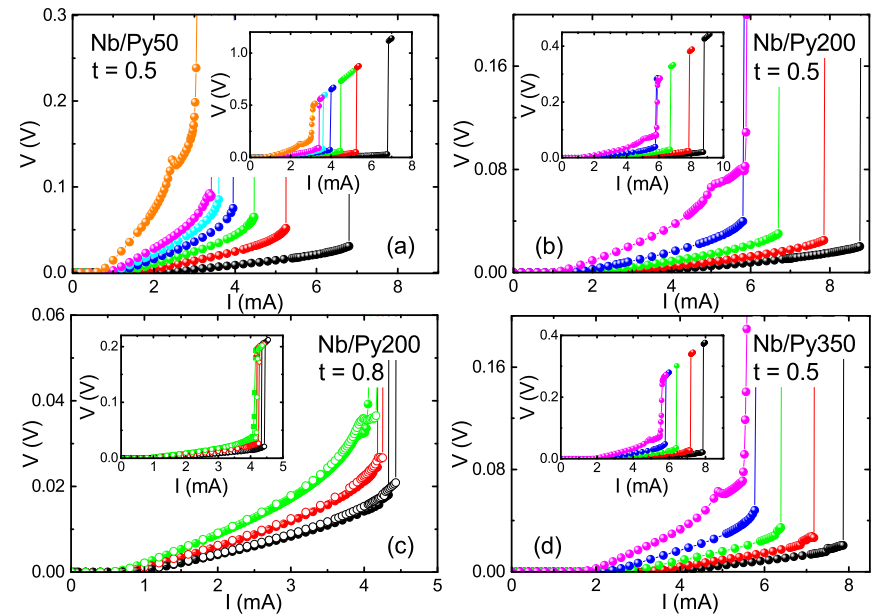

FIG. 2. (a) $I-V$ curves for the $\mathrm{Nb} / \mathrm{Py} 50$ bilayer at $t=0.5$ for different magnetic fields. From right to left, 0.01, 0.02, 0.03, 0.04, $0.05,0.06,0.13 \mathrm{~T}$. (b) $I-V$ curves for the $\mathrm{Nb} / \mathrm{Py} 200$ bilayer prepared in the $\mathrm{V}$ regime at $t=0.5$ for different magnetic fields. From right to left, $0.005,0.007,0.01,0.015,0.03 \mathrm{~T}$. (c) $I-V$ curves for the $\mathrm{Nb} / \mathrm{Py} 200$ bilayer at $t=0.8$ either in the $\mathrm{V}$ (closed symbols) or in the ESD (open symbols) regime for different magnetic fields. From right to left, $0.005,0.007,0.01$. (d) $I-V$ curves for the $\mathrm{Nb} / \mathrm{Py} 350$ bilayer prepared in the $\mathrm{V}$ regime at $t=0.5$ for different magnetic fields. From right to left, 0.005, 0.007, 0.01, 0.015, 0.025 T. The insets are a full-scale view of the $I-V$ curves shown in the main panels.

At high fields the jump is replaced by a more continuous transition towards the normal state, as is clearly seen looking at the curves corresponding to the fields of 0.4 and $0.5 \mathrm{~T}$ in the inset of Fig. 1. Correspondingly, we can define $\mu_{0} \widetilde{H}$ as the value of the field where the jump disappears. For all the above $I-V$ characteristics the flux-flow resistivity $\rho_{\mathrm{ff}}$ is consistently given by $\rho_{\mathrm{ff}}=\rho_{\mathrm{n}} H / H_{c 2 \perp}$.

Some $I-V$ characteristics for the $\mathrm{Nb} /$ Py bilayers are shown in the four panels of Fig. 2 for selected values of temperatures and fields. From the analysis of these curves it emerges that clear jumps at $V^{*}$ are present. In accordance with Ref. [6] the values of $v^{*}$ are higher with respect to the $\mathrm{Nb}$ film at the same reduced temperature. Similarly, $\mu_{0} \widetilde{H}$ is lower for the bilayers and scales both as a function of $d_{\text {Py }}$ and $t$. These experimental data confirm what was previously observed [2,6]; i.e., when the critical velocity is larger the instability typically disappears at smaller field and/or higher temperatures because in these cases the critical voltage $V^{*}$ is close to the normal state voltage $V_{\mathrm{n}}$. Indeed, at $t=0.5$ it is $\mu_{0} \widetilde{H}=0.4,0.13,0.03$, and $0.025 \mathrm{~T}$ for $\mathrm{Nb}, \mathrm{Nb} / \mathrm{Py} 50, \mathrm{Nb} / \mathrm{Py} 200$, and $\mathrm{Nb} / \mathrm{Py} 350$, respectively. These threshold values for the magnetic field decrease when the temperature is increased (the measurements are not shown here).

In Fig. 3 the magnetic field dependence of the critical current density $J_{\mathrm{c}}=I_{\mathrm{c}} /\left(w d_{\mathrm{Nb}}\right)$ at $t=0.5$ is shown for the three bilayers. $I_{\mathrm{c}}$ was determined by choosing a $5 \mu \mathrm{V}$ criterion. In agreement with the results previously reported for the same system [6], at low fields $J_{\mathrm{c}}$ is of the order of $10^{10} \mathrm{~A} / \mathrm{m}^{2}$ and one order of magnitude lower than the values measured for the single $\mathrm{Nb}$ film (not shown here). Moreover, the $J_{\mathrm{c}}$

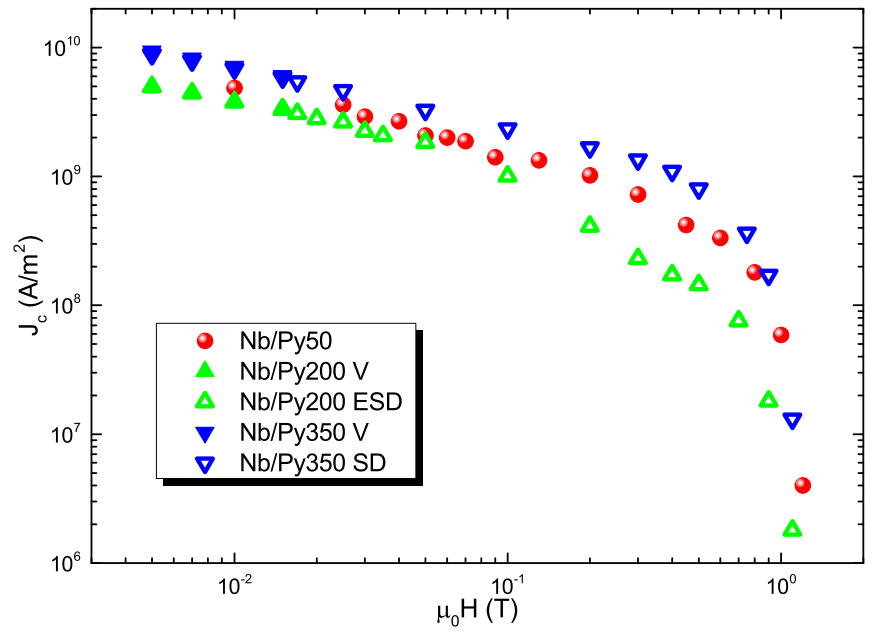

FIG. 3. Critical current density as a function of the magnetic field at $t=0.5$ for the three bilayers. In the case of $\mathrm{Nb} / \mathrm{Py} 200$ and $\mathrm{Nb} / \mathrm{Py} 350$ open symbols refer to the ESD and SD state, respectively, while closed symbols show the measurements for both samples prepared in the $\mathrm{V}$ regime.

values are almost independent of $d_{\mathrm{Py}}$ and, in the case of $\mathrm{Nb} / \mathrm{Py} 200$ and $\mathrm{Nb} / \mathrm{Py} 350$, they are not strongly influenced by the inhomogeneity of the magnetic phase of the Py layers, as also shown in panel (c) of Fig. 2 where, for voltage values lower than $V^{*}$, the two curves overlap for the three values of the magnetic field.

\section{DISCUSSION}

After checking the consistency of the main results concerning the values of $v^{*}, \mu_{0} \widetilde{H}$, and $J_{\mathrm{c}}(H)$ in the case of the single $\mathrm{Nb}$ film and $\mathrm{Nb} / \mathrm{Py}$ bilayers (with Py being in the $\mathrm{V}$ regime), we now focus on the main aim of this work, namely the influence of the magnetic configuration on the critical velocity of the vortex lattice. For this purpose, the behavior of the $\mathrm{Nb} / \mathrm{Py} 200$ and $\mathrm{Nb} / \mathrm{Py} 350$ bilayers was investigated by comparing the $I-V$ acquired in the virgin state and after inducing the inhomogeneous state in the samples: ESD and $\mathrm{SD}$, respectively. Indeed, the curves measured at the same field for the samples prepared in the two different regimes behave differently at the instability point. As an example, in panel (c) of Fig. 2 we show the $I-V$ curves for the bilayer Nb/Py200 at $t=0.8$ both in the $\mathrm{V}$ and in the ESD regime for three different fields.

Before starting the analysis of the flux-flow instability at the critical velocity, it is relevant to rule out the possibility that the sudden jump to the normal state at $I=I^{*}$ is due to Joule heating of the strip. The Bezuglyj and Shklovskij (BS) theory [39] predicts a threshold value $H_{\mathrm{T}}$ for the magnetic field above which the thermal effect starts to have a considerable role in the dynamics of the vortices. This value can be obtained by fitting the dissipated power $P^{*}=I^{*} V^{*}$ at the instability point as function of $H / H_{\mathrm{T}}$ through a well defined function, i.e., $P^{*}=P_{0}(1-a)$, where $a=\left[1+b+\left(b^{2}+8 b+4\right)^{1 / 2}\right] /[3(1+2 b)]$ and $b=H / H_{\mathrm{T}}$ [39]. In Fig. 4(a) we show the magnetic field dependence of the dissipated power at the instability point at $t=0.5$ for 


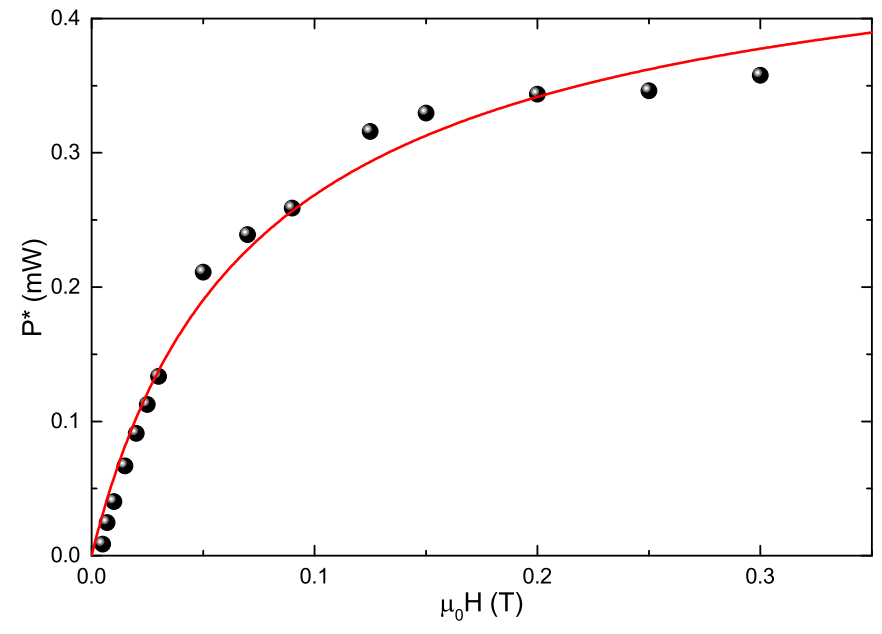

FIG. 4. Dissipated power at the instability point as a function of the magnetic field at $t=0.5$ for the single $\mathrm{Nb}$ film. The solid line is the fit to the data according to the BS theory (see text).

the $\mathrm{Nb}$ film together with the fit to the data obtained using the above expression. The result extracted from the fit gives $\mu_{0} H_{\mathrm{T}}=0.15 \mathrm{~T}$. In the case of the bilayers, the values obtained for $\mu_{0} H_{\mathrm{T}}$ are always of the order of $\mu_{0} \widetilde{H}$. This implies that all the data over which the analysis of critical velocity was performed are not affected by thermal effects.

The critical velocities $v^{*}$ as determined from the measured values of the critical voltage $V^{*}$ for the $\mathrm{Nb}$ film and for the $\mathrm{Nb} /$ Py50 bilayer are shown in Fig. 5 as a function of the field at $t=0.5$ together with the data referring to the bilayer $\mathrm{Nb} / \mathrm{Py} 200$ in the $\mathrm{V}$ regime. The anomalous increase of $v^{*}$ at very low fields (up to $\sim 0.05 \mathrm{~T}$ ) followed by a decrease at higher fields obtained for the $\mathrm{Nb}$ film confirms what was already observed in thin films of superconducting materials in the dirty limit and in the nonmesoscopic regime [34-38,40]. The value $v^{*} \simeq 600 \mathrm{~m} / \mathrm{s}$ at high field is larger with respect to what was previously measured in $\mathrm{Nb}$ single films at similar fields and temperatures $[2,6,7]$. This result can be ascribed to

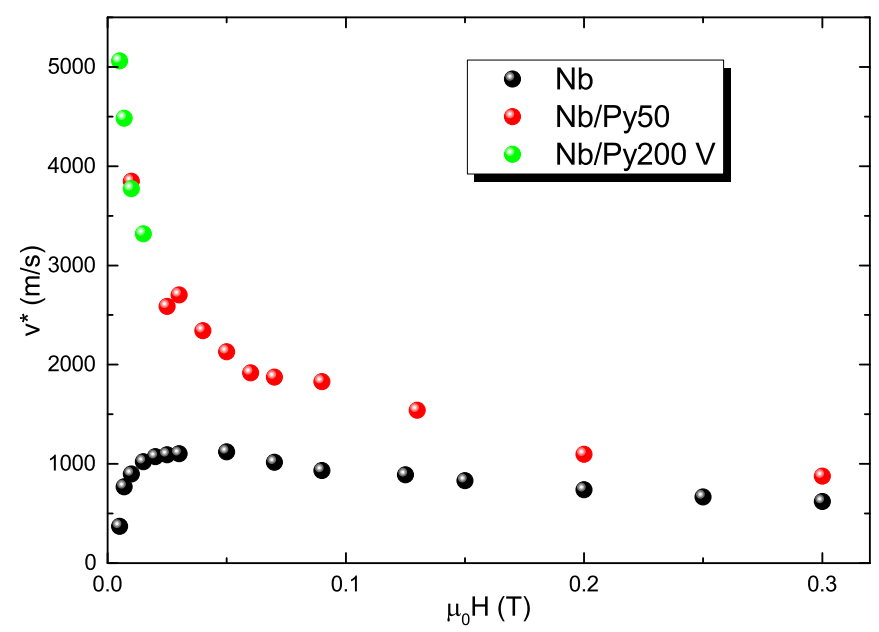

FIG. 5. Critical velocity $v^{*}$ versus the applied magnetic field at $t=0.5$ for the $\mathrm{Nb}$ single film, the $\mathrm{Nb} / \mathrm{Py} 50$, and the $\mathrm{Nb} / \mathrm{Py} 200$ in the $\mathrm{V}$ regime.

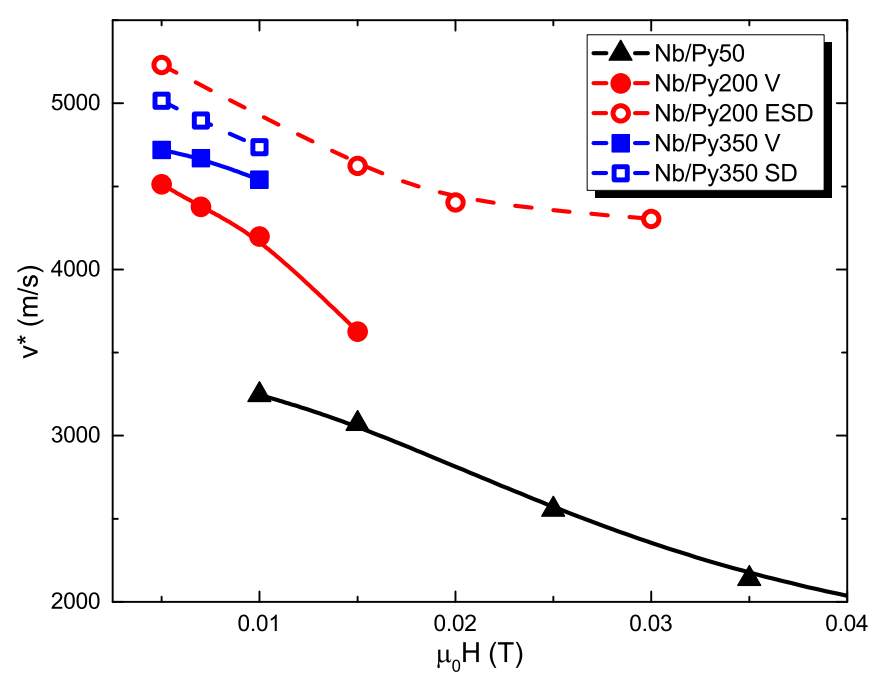

FIG. 6. $v^{*}$ versus field at $t=0.8$ for the $\mathrm{Nb} / \mathrm{Py} 50$ and for the $\mathrm{Nb} / \mathrm{Py} 200$ and $\mathrm{Nb} / \mathrm{Py} 350$ bilayers prepared either in the virgin or in inhomogeneous magnetic state. The solid lines are guides to the eye.

the larger resistivity of the $\mathrm{Nb}$ under study, since disorder leads to an appreciable increase of the critical velocity [26]. On the other hand, looking at the data of the two Nb/Py bilayers, it is clear that the presence of the ferromagnetic layer suppresses the nonmonotonic behavior of $v^{*}$ at low fields and gives larger values of the critical velocity. In particular, for the $\mathrm{Nb} / \mathrm{Py} 50$ it is $v^{*} \simeq 1000 \mathrm{~m} / \mathrm{s}$, a value already obtained at a comparable reduced temperature at the saturation field for $\mathrm{Nb} / \mathrm{Py}$ bilayers with $d_{\mathrm{Py}}$ such that $\mathrm{Py}$ is in the $\mathrm{H}$ regime [6].

More interestingly, the presence of the magnetic inhomogeneity affects the values of the critical velocities. In Fig. 6 the magnetic field dependence of $v^{*}$ at $t=0.8$ is shown for the $\mathrm{Nb} / \mathrm{Py} 200$ and the $\mathrm{Nb} / \mathrm{Py} 350$ bilayers both in the $\mathrm{V}$ regime (closed symbols) and in the magnetic inhomogeneous (open symbols) regime. In the same figure data for the NbPy50 bilayer are also reported. In particular, in the (E)SD regimes the values of $v^{*}$ are larger with respect to the $\mathrm{V}$ regime, with an increase which is more pronounced for the $\mathrm{Nb} / \mathrm{Py} 200$. In both bilayers this effect is more relevant close to $T_{\mathrm{c}}$, probably because at these temperatures the superconducting order parameter, which is already reduced, is more strongly influenced by the perturbation induced by the inhomogeneous out-of-plane component of the magnetization present in the ESD and SD regimes [10]. All these values are larger than the critical velocities obtained for the $\mathrm{Nb} / \mathrm{Py} 50$. Moreover, is worthwhile to notice that the values of $v^{*} \gtrsim 5000 \mathrm{~m} / \mathrm{s}$ measured at $t=0.8$ at low fields are substantially larger than those previously reported on $\mathrm{S} / \mathrm{F}$ bilayers based on $\mathrm{Nb}$ [2].

Finally, from the obtained values of $v^{*}$ and the quasiparticle diffusion coefficient $D$ evaluated for the single $\mathrm{Nb}$ film $[6,41,42]$, by using the expression [9]

$$
v^{*}=\frac{D^{1 / 2}[14 \zeta(3)]^{1 / 4}(1-t)^{1 / 4}}{\left(\pi \tau_{\mathrm{E}}\right)^{1 / 2}}
$$

we estimated the values for the relaxation time of the quasiparticles, $\tau_{\mathrm{E}}$. The values of $\tau_{\mathrm{E}}$ at the reduced temperature $t=0.8$, namely when we expect a stronger influence of the magnetic configuration on the relaxation process, are summarized in 
TABLE II. Quasiparticle relaxation time for the three bilayers calculated at $\mu_{0} H=0.01 \mathrm{~T}$ and $t=0.8$. For the samples $\mathrm{Nb} / \mathrm{Py} 200$ and $\mathrm{Nb} / \mathrm{Py} 350$ numbers refer either to the virgin or to inhomogeneous magnetic state.

\begin{tabular}{lc}
\hline \hline Sample & $\tau_{\mathrm{E}}(\mathrm{s})$ \\
\hline $\mathrm{Nb} /$ Py50 & $1.00 \times 10^{-11}$ \\
$\mathrm{Nb} /$ Py200 V & $5.80 \times 10^{-12}$ \\
$\mathrm{Nb} /$ Py200 ESD & $4.80 \times 10^{-12}$ \\
$\mathrm{Nb} /$ Py350 V & $4.93 \times 10^{-12}$ \\
$\mathrm{Nb} /$ Py350 SD & $4.53 \times 10^{-12}$ \\
\hline \hline
\end{tabular}

Table II. As a consequence of the enhancement of $v^{*}$ in the presence of the magnetic inhomogeneity, the corresponding values of $\tau_{\mathrm{E}}$ for the $\mathrm{Nb} / \mathrm{Py} 200$ and the $\mathrm{Nb} / \mathrm{Py} 350$ in the ESD and SD state, respectively, assume lower values compared to the homogeneous state. We believe that this result is due to the presence of an inhomogeneous magnetization in the Py layers, which does not affect the pinning properties (see Fig. 3) but seems to induce a further reduction of the relaxation time. Furthermore, the values of $\tau_{\mathrm{E}}$ are almost a factor of 2 smaller than those obtained for the sample $\mathrm{Nb} / \mathrm{Py} 50$. However, the very small values obtained for $\tau_{\mathrm{E}}$, in both cases of the order of $10^{-12} \mathrm{~s}$, can also be related to the dirty nature of our $\mathrm{Nb}$ films, since it is known that the relaxation time is strongly influenced by the disorder present in the system [2,26]. As discussed in Refs. [2,8] the coupling with a disordered ferromagnet may be responsible of a change in the dominant relaxation process, from electron-electron $(e e)$ recombination to electron-phonon (ep) scattering. Indeed, it is known that the total relaxation time of the quasiparticles is the parallel sum of each relaxation time relative to a different scattering channel and so, in the most common case, $\tau_{\mathrm{E}}=\left(\tau_{\mathrm{ee}}^{-1}+\tau_{\mathrm{ep}}^{-1}\right)^{-1}$, where $\tau_{\mathrm{ee}}$ and $\tau_{\mathrm{ep}}$ are respectively the times for $e e$ recombination and $e p$ scattering. Taking into account the reduction of $\tau_{\mathrm{E}}$ caused by the ESD or $\mathrm{SD}$ regimes and the previous consideration, it can be said that the change in the magnetic configuration mainly influences the ep relaxation channel. To understand if this change makes the $e p$ scattering the prevalent relaxation mechanism it could be useful to investigate in clean samples the temperature dependence of the quasiparticle relaxation time to obtain additional information on the inelastic scattering mechanisms present in the hybrids $[2,43]$.

\section{CONCLUSIONS}

In conclusion, by measuring $I-V$ characteristics we studied the dynamic instability of the vortex lattice in $\mathrm{Nb} / \mathrm{Py}$ bilayers. The Py thickness was chosen in order to tune their magnetic state. Once again, we find clear fingerprints of the presence of a magnetically inhomogeneous state well below the transition to the stripe domain phase. Already in the ESD phase, the critical velocities for the vortex lattice instability are well above what is found for the homogeneous magnetic regime. From the magnetic field dependence of the critical velocity for the onset of the vortex motion we estimated the values of the quasiparticle relaxation time. In the case of the thicker samples this value is almost a factor of 2 smaller than the one estimated for the sample with a magnetically homogeneous Py layer $\left(d_{\mathrm{Py}}<d_{\mathrm{cr}}^{\mathrm{ESD}}\right)$. These results confirm once more that the quasiparticle relaxation processes are strongly affected by the nature and the thickness of the proximized ferromagnetic layer. Finally, a further reduction of $\tau_{E}$ was observed in the ESD and SD states. This last result may deserve further investigation, in view of the recent evidence of an anomalous long range proximity effect in $\mathrm{Nb} / \mathrm{Py}$ hybrids, induced by a magnetically inhomogeneous permalloy layer, in order to clarify if this reduction can be connected to the generation of a new relaxation channel [16].

\section{ACKNOWLEDGMENTS}

S.V. and J.A. acknowledge support from the Netherlands Organisation for Scientific Research (NWO). The work was also supported by the EU COST action MP1201 "NanoSC".
[1] C. Cirillo, V. Pagliarulo, H. Myoren, C. Bonavolontà, L. Parlato, G. P. Pepe, and C. Attanasio, Phys. Rev. B 84, 054536 (2011).

[2] C. Attanasio and C. Cirillo, J. Phys.: Condens. Matter 24, 083201 (2012).

[3] T. Taneda, G. P. Pepe, L. Parlato, A. A. Golubov, and R. Sobolewski, Phys. Rev. B 75, 174507 (2007).

[4] D. Pan, G. P. Pepe, V. Pagliarulo, C. De Lisio, L. Parlato, M. Khafizov, I. Komissarov, and R. Sobolewski, Phys. Rev. B 78, 174503 (2008).

[5] N. Marrocco, G. P. Pepe, A. Capretti, L. Parlato, V. Pagliarulo, G. Peluso, A. Barone, R. Cristiano, M. Ejrnaes, A. Casaburi, N. Kashiwazaki, T. Taino, H. Myoren, and R. Sobolewski, Appl. Phys. Lett. 97, 092504 (2010).

[6] A. A. Armenio, C. Bell, J. Aarts, and C. Attanasio, Phys. Rev. B 76, 054502 (2007).

[7] C. Cirillo, E. A. Ilyina, and C. Attanasio, Supercond. Sci. Technol. 24, 024017 (2011).
[8] E. A. Ilyina, C. Cirillo, and C. Attanasio, Eur. Phys. J. B 83, 53 (2011).

[9] A. I. Larkin and Yu. N. Ovchinnikov, Sov. Phys. JETP 41, 960 (1976).

[10] S. Voltan, C. Cirillo, H. J. Snijders, K. Lahabi, A. GarcíaSantiago, J. M. Hernández, C. Attanasio, and J. Aarts, Phys. Rev. B 94, 094406 (2016)

[11] N. Saito, H. Fujiwara, and Y. Sugita, J. Phys. Soc. Jpn. 19, 421 (1964).

[12] N. Amos, R. Fernandez, R. Ikkawi, B. Lee, A. Lavrenov, A. Krichevsky, D. Litvinov, and S. Khizroev, J. Appl. Phys. 103, 07E732 (2008).

[13] A. Belkin, V. Novosad, M. Iavarone, J. Fedor, J. E. Pearson, A. Petrean-Troncalli, and G. Karapetrov, Appl. Phys. Lett. 93, 072510 (2008).

[14] J. Ben Youssef, N. Vukadinovic, D. Billet, and M. Labrune, Phys. Rev. B 69, 174402 (2004). 
[15] T. Dastagir, W. Xu, S. Sinha, H. Wu, Y. Cao, and H. Yu, Appl. Phys. Lett. 97, 162506 (2010).

[16] C. Cirillo, S. Voltan, E. A. Ilyina, J. M. Hernández, A. GarcíaSantiago, J. Aarts, and C. Attanasio, New J. Phys. 19, 023037 (2017).

[17] F. S. Bergeret, A. F. Volkov, and K. B. Efetov, Phys. Rev. Lett. 86, 4096 (2001).

[18] M. Eschrig, Phys. Today 64(1), 43 (2011).

[19] J. Guimpel, M. E. de la Cruz, F. de la Cruz, H. J. Fink, O. Laborde, and J. C. Villègier, J. Low Temp. Phys. 63, 151 (1986).

[20] P. R. Broussard, Phys. Rev. B 43, 2783 (1991).

[21] Handbook of Chemistry and Physics, edited by R. C. Weast (The Chemical Rubber Co., Cleveland, 1972).

[22] H. R. Kerchner, D. K. Christen, and S. T. Sekula, Phys. Rev. B 24, 1200 (1981).

[23] W. Klein, R. P. Huebener, S. Gauss, and J. Parisi, J. Low Temp. Phys. 61, 413 (1985).

[24] A. V. Samoilov, M. Konczykowski, N.-C. Yeh, S. Berry, and C. C. Tsuei, Phys. Rev. Lett. 75, 4118 (1995).

[25] C. Villard, C. Peroz, and A. Sulpice, J. Low Temp. Phys. 131, 957 (2003).

[26] C. Peroz and C. Villard, Phys. Rev. B 72, 014515 (2005).

[27] S. G. Doettinger, R. P. Huebener, R. Gerdemann, A. Kühle, S. Anders, T. G. Träuble, and J. C. Villegier, Phys. Rev. Lett. 73, 1691 (1994).

[28] S. G. Doettinger, S. Kittelberger, R. P. Huebener, and C. C. Tsuei, Phys. Rev. B 56, 14157 (1997).

[29] Z. L. Xiao, P. Voss-de Haan, G. Jakob, and H. Adrian, Phys. Rev. B 57, R736(R) (1998).
[30] Z. L. Xiao, P. Voss-de Haan, G. Jakob, Th. Kluge, P. Haibach, H. Adrian, and E. Y. Andrei, Phys. Rev. B 59, 1481 (1999).

[31] B. J. Ruck, J. C. Abele, H. J. Trodahl, S. A. Brown, and P. Lynam, Phys. Rev. Lett. 78, 3378 (1997).

[32] B. J. Ruck, H. J. Trodahl, J. C. Abele, and M. J. Geselbracht, Phys. Rev. B 62, 12468 (2000).

[33] C. Peroz, C. Villard, A. Sulpice, and P. Butaud, Physica C 222-226, 369 (2002).

[34] G. Grimaldi, A. Leo, A. Nigro, S. Pace, C. Cirillo, and C. Attanasio, Physica C 468, 765 (2008).

[35] G. Grimaldi, A. Leo, A. Nigro, S. Pace, A. A. Armenio, and C. Attanasio, J. Phys.: Conf. Ser. 97, 012111 (2008).

[36] G. Grimaldi, A. Leo, A. Nigro, S. Pace, and R. P. Huebener, Phys. Rev. B 80, 144521 (2009).

[37] G. Grimaldi, A. Leo, C. Cirillo, C. Attanasio, A. Nigro, and S. Pace, J. Phys.: Condens. Matter 21, 254207 (2009).

[38] G. Grimaldi, A. Leo, D. Zola, A. Nigro, S. Pace, F. Laviano, and E. Mezzetti, Phys. Rev. B 82, 024512 (2010).

[39] A. I. Bezuglyj and V. A. Shklovskij, Physica C 202, 234 (1992).

[40] G. Grimaldi, A. Leo, C. Cirillo, A. Casaburi, R. Cristiano, C. Attanasio, A. Nigro, S. Pace, and R. P. Huebener, J. Supercond. Nov. Magn. 81, 24 (2011).

[41] Z. Radovic, L. Dobrosavljevic-Grujic, A. I. Buzdin, and J. R. Clem, Phys. Rev. B 38, 2388 (1988).

[42] P. Koorevaar, Y. Suzuki, R. Coehoorn, and J. Aarts, Phys. Rev. B 49, 441 (1994).

[43] A. Leo, G. Grimaldi, R. Citro, A. Nigro, S. Pace, and R. P. Huebener, Phys. Rev. B 84, 014536 (2011). 